Bemerkungen über das Integral $\Gamma(a)$.

Von

L. Pochrammer in Kiel.

\title{
\& 1.
}

Durch $\bar{\Gamma}(a)$ werde, wie in der Abbandlung des Verfassers ,"Zur Theorie der Euler'schen Integrale " im $35^{\text {ten }}$ Bande dieser Annaleu $(\S 3$, S. 514), das Integral

$$
\bar{\Gamma}(a)=\int_{-\infty}^{\bar{c}(0)} e^{u} u^{a-1} d u
$$

bezeichnet, in welchem die Variable $u$, vom unendlich entfernten Punkte der negativen reellen Axe ausgehend und dahin zurilckkehrend, einen positiven Umlauf um den Punkt 0 ausführt*). Für $\widetilde{\Gamma}(a)$ kann (S. 515 des genannten $35^{\text {ten }}$ Bandes) auch der Ausdruck

$$
\bar{\Gamma}(a)=e^{-\pi i \alpha} \int_{+\infty}^{(0)} e^{-n} u^{a-1} d u,
$$

wo der Integrationsweg einen bei $+\infty$ beginnenden positiven Umlauf um den Punkt 0 darstellt, oder der Ausdruck**)

$$
\bar{\Gamma}(a)=-e^{\pi i a} \bar{\int}_{+\infty}^{(0-)} e^{-u} u^{a-1} d u
$$

in welchem die Umkreisung des Punktes 0 in negativer Drehungsrichtung erfolgt, gesetzt werden. Die Formel (3) entsteht aus der Formel (2) durch Umkehrung des Integrationsweges. Die Potenz $u^{a-1}$

-) Das Integral $\bar{\Gamma}(a)$ weicht nur in formaler Bezichung von dem Integral $\Omega b$, welches $\mathrm{H}$. Hankel in $\$ \mathbf{3}$ seiner $\mathbf{A b h}$. ,Die Euler'schen Integrale bei unbeschränkter Variabilität des Arguments", Schlömilch's Zeitschr. für Math. u. Phys., Jahrgang 9, 1864, definirt hat.

*) Cfr. die Abh. des Verf. „, Oeber einige besondere Fälle der linearen Differentialgleichung $2^{\text {ter }}$ Ordnung mit linearen Coefficienten", Band 38 dieser Annslen, S. 243. 
wird bei dem Integral (1) dadurch näher bestimmt, dass im Schnittpunkte des Integrationsweges mit der positiven reellen Axe $u^{a-1}$ gleich der Grösse $e^{(a-1) \log \imath}$, in welcher log $u$ den reellen Logarithmus bedeutet, zu nehmen ist. Letztere Bedingung gilt in (2) und (3) für den ersten Theil des Integrationsweges.

Die obigen Integrale sollen, mit Rücksicht auf gewisse Anwendungen, durch die Substitution

$$
u=\frac{1}{t}, u^{a-1} d u=-t^{a-1} d t,
$$

umgeformt werden. Der Weg der Variable $t$ nimmt daun im Punkte 0 seinen Anfang, und zwar verlässt $t$ bei dem Integral (1) den Nullpunkt in der Richtung der negativen reellen Axe, bei den Integralen (2) und (3) in der Richtung der positiven reellen Axe. Man setze der Einfachheit halber den Weg der Variable $u$ aus einem (doppelt durchlaufenen) Abschnitt der negativen, bezw. positiven reellen Axe und aus einem Kreise, der um den Nullpunkt mit dem Radius 1 beschrieben wird, zusammen. Unter $e$ werde eine unendlich kleine positive reelle Constante verstanden. Dann besteht der Weg, der sich für $t$ bei dem Integral (1) ergiebt, aus der geradlinigen Strecke rom Punkte - $\mathfrak{c}$ bis zum Punkte -1 , dem im negativen Sinne durchlaufenen Kreise mit dem Radius 1, und der geradlinigen Strecke von -1 bis Dieser Weg stellt einen rom Punkte $-\mathfrak{c}$ aus bewirkten negativen Umlauf um den Punkt 0 dar. Unter Benutzung der in $\$ 1$ der Abhandlung des Verfassers ,Ueber ein Integral mit doppeltem Unlauf ${ }^{6 *}$ )

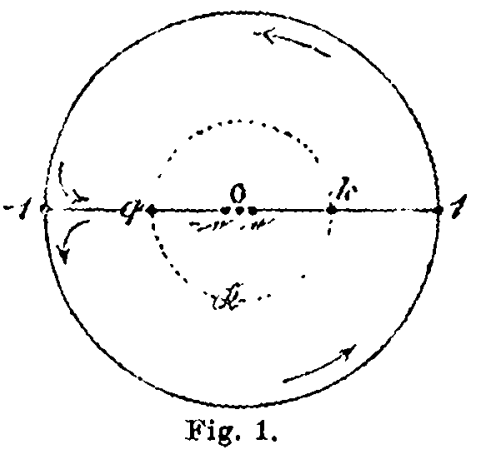
angegebenen Bezeichnung (die auch in (1), (2), (3) angewendet ist) erhält man demnach die Gleichung

$$
\bar{\Gamma}(a)=-\int_{-\varepsilon}^{10-1} e^{\frac{1}{t}} t^{-a-1} d t .
$$

Man kann nun unter Hinzufügung des Factors - 1 den Integrationsweg umkebren. Dies indert hier den Weg nur insofern, als die negative Umkreisung des Nullpunktes sich in die positive verwandelt (Fig. 1). Also ergiebt sich, unter der $\mathrm{Be}$ dingung, dass für $t=1$ die Potenz $t^{-a-1}$ gleich 1 gesetzt werde, die Formel

$$
\tilde{r}(a)=\int_{-\varepsilon}^{(\omega)} e^{\frac{1}{t}} t^{-a-1} d t
$$

Die Substitution (4) lässt aus (3) die Gleichung 
und aus (2) die Gleichung

$$
\bar{\Gamma}(a)=e^{\pi i a} \int_{\mathrm{e}}^{\bar{c}(0)} e^{-\frac{1}{t}} t^{-a-1} d t
$$

$$
\bar{\Gamma}(a)=-e^{-\pi i a} \int_{\mathrm{e}}^{(0-)} e^{-\frac{1}{t}} t^{-\alpha-1} d t
$$

entstehen, woselbst $\mathfrak{e}$, wie oben, eine unendlich kleine positive reelle Zahl bedeutet. In (6) und (7) nimmt die Potenz $t^{-a-1}$ den Werth 1 an, wenn $t$ zum ersten Male im Punkte 1 eintrifft.

Statt des Kreises nuit dem Radius 1 kann in (5), (6), (7), als Theil des Weges von $t$, ein Kreis $\mathfrak{\Omega}$ gewählt werden, der mit einem beliebigen Radius $k$ um den Nullpunkt gelegt wird. Denn die zu integrirenden Functionen haben keinen anderen endlichen singulären Punkt als den Punkt $t=0$. Ausser dem Kreise $\Re$ bildet dann in (5) die (in beiden Richtungen vorkommende) Strecke von - $\mathrm{e}$ bis $-k$, und in (6) und (7) die Strecke von $+\mathfrak{c}$ bis $+k$ den Integrationsweg. Es gilt in diesem Falle für das Integral (5) die Bestimmung, dass $t^{-a-1}$ im Punkte $t=\pi$ gleich $e^{-(a+1) \log k}$ wird, wo $\log 7$ den reellen Logarithmus bezeichnet. Bei den Integralen (6) und (7) stimmt $t^{-a-1}$ auf dem ersten Theile des Integrationsweges (zwischen $\mathfrak{c}$ und $k$ ) mit dem Werthe $e^{-(a+1) \log t}$, in welchem $\log t$ reell ist, überein.

\section{$\S 2$.}

In dem Integral (1) wurde der unendlich entfernte Punkt der negativen reellen Axe als Ausgangspunkt und Endpunkt des Integrationsweges angenommen. Indessen kann man diesen Punkt, ohne dass der Werth des Integrals sich änderte, durch einen beliebigen Punkt derjenigen Hälfte des unendlichen Horizontes, bei der die reellen Bestandtheile negativ sind, ersetzen, resp. zwei solche Horizontpunkte als untere und obere Begrenzung des Integrals nach $u$ wählen. Dies folgt daraus, dass, wie Cauchy gezeigt hat, das Integral der Function $e^{u} u^{a-1}$, genommen über irgend einen Theil der genannten Hälfte des Horizonts, einen verschwindend kleinen Werth hat.

Wird unter $s$ eine sehr grosse positive reelle Constante verstanden, und $u=s e^{\vartheta i}$ gesetzt, so ergiebt sich, wenn

$$
a=a_{1}+i a_{2}
$$

ist $\left(a_{1}, a_{2}\right.$ reell), die Gleichung

$$
e^{u} u^{a-1} d u=e^{s(\cos \vartheta+i \sin \vartheta)} s^{a_{1}} e^{i a_{2} \log s} \mathrm{e}^{\left(i a_{1}-a_{2}\right) \vartheta} i d \vartheta .
$$

Man nehme, indem man durch $\lambda$ eine reelle, zwischen 0 und $\frac{\pi}{2}$ liegende Constante und durch $s^{\prime}$ das Product $s e^{-(\pi-\lambda) i}$ bezeichnet, die Werthe 
$-\pi$ und $-(\pi-\lambda)$ als Grenzen der Integration nach $\vartheta$, so dass $-s$ und $s$ die Integralgrenzen in Bezug auf $u$ sind. Da

$$
\bmod \left(e^{u} u^{a-1} d u\right)=s^{a_{1}} e^{s \cos \vartheta-a_{2} \vartheta} d \vartheta
$$

ist, so besteht für den Modul des betrachteten Integrals die Ungleichbeit

Hieraus folgt

$$
\bmod . \int_{-s}^{s^{\prime}} e^{u} u^{\alpha-1} d u<s^{a_{1}} \int_{-\pi}^{-(\pi-\lambda)} e^{s \cos \vartheta-\alpha_{2} \vartheta} d \vartheta
$$

$$
\bmod \int_{-s}^{s^{\prime}} e^{u} u^{a-1} d u<\lambda s^{a_{1}} e^{s \cos \vartheta_{0}-\alpha_{2} \vartheta_{s}}
$$

wo $\vartheta_{0}$ eine Constante bedeutet, die zwischen $-\pi$ und $-(\pi-\lambda)$ liegt. Mit unbegrenzt wachsendem $s$ wird aber, da $\cos \vartheta_{0}$ negativ ist, die rechte Seite von (8) beliebig klein, was um so mehr ron dem links stehenden Integrale gilt. In derselben Art wird bewiesen, dass das Integral nach $\vartheta$ zwischen den Grenzen $-(\pi+\lambda)$ und $-\pi$ oder, was auf dasselbe binauskommt, zwischen den Grenzen $\pi-\lambda$ und $\pi$ sich für ein unendliches $s$ dem Werthe Null nähert.

Man ziehe nun rom Nullpunkte aus zwei Gerade $\mathfrak{M}_{1}$ und $\mathfrak{M}_{2}$, welche mit der positiven reellen Axe die Winkel $-\left(\pi-\lambda_{1}\right)$ und $\pi-\lambda_{2}$

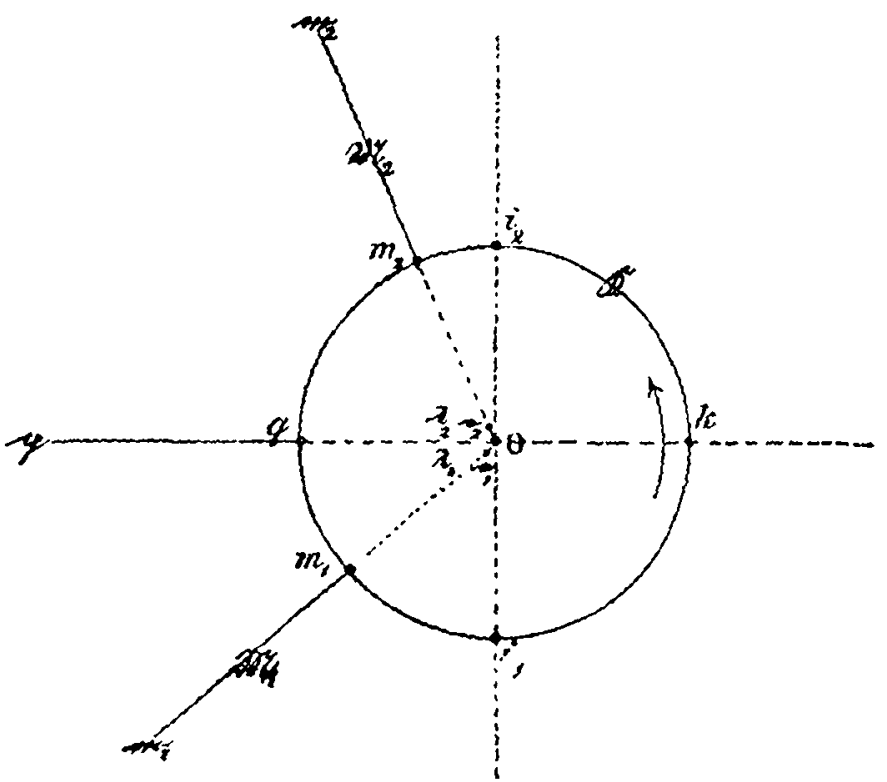

Fig. 2 . bilden; $\lambda_{1}$ und $\lambda_{2}$ seien positiv und kleiner als $\frac{\pi}{2}$. Die unendlich entfernten Punkte der Geraden $\mathfrak{M}_{1}, \mathfrak{M}_{2}$ sollen kurz durch $\mathfrak{m}_{1}, \mathfrak{m}_{2}$, und der unendlich entfernte Punktder negativen reellen Axe durch bezeichnet werden. Ferner möge der Kreis $\Re$, der mit dem beliebigen Radius $k$ um den Nullpunkt beschrieben wird, die Geraden $\mathfrak{M}_{1}, \mathfrak{M}_{2}$ in den Punkten $m_{1}, m_{2}$ und die negative reelle Axe im Punkte $q$ treffen (Fig. 2). Die Schnittpunkte des Kreises mit der positiven reellen Axe, mit der negativen imaginären und der positiven imaginären Axe werden $k, i_{1}$ und $i_{2}$ genannt.

In dem Integrationswege des Integrals (1), welcher nach Fig. 2 aus den Theilen

$$
q q, q m_{1} i_{1} k i_{2} m_{2} q, q \dot{\mathfrak{q}}
$$

besteht, dürfen die Strecken $q q$ und $q m_{1}$ durch den Bogen $q m_{1}$ des 
unendlichen Horizonts und den geradlinigen Abschnitt $\mathfrak{m}_{1} m_{1}$ ersetzt werden. Denn das Integral von $e^{u} u^{a-1}$ längs des geschlossenen Weges $q q, q m_{1}, m_{1} \mathfrak{m}_{1}, m_{1} q$ verschwindet, da die genannte Function auf dem eingeschlossenen Flächenstück eindeutig und stetig ist. Ebenso kann man statt $m_{2} q$ und $q q$ die Strecken $m_{2} m_{2}$ und $m_{2} \uparrow$ wählen. Die Integration von $e^{u} u^{a-1}$ längs der Bögen $\mathfrak{q} \mathfrak{m}_{1}$ und $\mathfrak{m}_{2} \mathfrak{q}$ liefert aber, wie gezeigt wurde, das Resultat Null. Also ist $\bar{\Gamma}(a)$ auch gleich dem Integral

$$
\bar{\Gamma}(a)=\int_{\mathfrak{m}_{1}}^{\mathfrak{m}_{2}} e^{u} u^{a-1} d u,
$$

dessen Grenzen $\mathfrak{m}_{1}, \mathfrak{m}_{2}$ als die Ausdrücke

$$
\mathfrak{m}_{1}=\lim _{(s=\infty)} s e^{-\left(\pi-\lambda_{1}\right) i}, \quad \mathfrak{m}_{2}=\lim _{(s=\infty)} s e^{\left(\pi-\lambda_{2}\right) i}
$$

$\left(0 \leqq \lambda_{1}<\frac{\pi}{2}, 0 \leqq \lambda_{2}<\frac{\pi}{2}\right)$ definirt sind, und dessen Integrationsweg sich aus den Strecken

$$
\mathrm{m}_{1} m_{1}, m_{1} i_{1} k i i_{2} m_{2}, m_{2} m_{2}
$$

zusanmensetzt. Fügt man zu dem Integral (9) das (unendlich kleine) Integral der Function $e^{u} u^{\alpha-1}$ längs des Bogens $\varkappa_{2} q m_{1}$ des Horizonts hinzu und transformirt den Weg in der angegebenen Art, so hat man die Formel

$$
\bar{\Gamma}(a)=\int_{m_{1}}^{(0)} e^{u} u^{a-1} d u,
$$

in welcher für die Variable $u$ (nach Fig. 2) die geschlossene Curve

$$
m_{1} m_{1} i_{1} k i_{2} m_{2} q m_{1} m_{1}
$$

zur Anwendung kommt. Statt $\mathfrak{m}_{1}$ kann in (11) auch der Punkt $\mathfrak{m}_{2}$ zum Ausgangspunkt der Integration genommen werden; in diesem Falle durchläuft $u$ den Weg

$$
\mathfrak{m}_{2} m_{2} q m_{1} i_{1} k i_{2} m_{2} \mathfrak{m}_{2} \text {. }
$$

Für die Potenz $u^{a-1}$ gilt in (9) und (11) dieselbe Bestimmung wie in (1).

Die obigen Betrachtungen übertragen sich auf die Integrale (2) und (3). Integrirt man die Function $e^{-u} u^{a-1}$ längs eines Abschnittes derjenigen Hälfte des unendlichen Horizonts, wo die reellen Bestandtheile positiv sind, so ist das Integral unendlich klein. Also kann man in (2) und (3) den Weg der Variable $u$ in der Art abändern, dass die Integralgrenzen nicht in den unendlich entfernten Punkt der positiven reellen Axe, sondern in beliebige andere Punkte der soeben bezeichneten Horizonthälfte fallen. Aus (2) werden auf diese Weise die Gleichungen

$$
\bar{\Gamma}(a)=c^{-\pi i a} \int_{\mathfrak{p}_{1}}^{\mathfrak{p}_{2}} e^{-u} u^{a-1} d u
$$

und 


$$
\bar{\Gamma}(a)=e^{-\pi i a} \tilde{\int}_{p^{\prime}}^{(0)} e^{-u} u^{a-1} d u
$$

abgeleitet, in denen

$$
p_{1}=\lim _{(s=\infty)} s e^{\lambda_{1} i}, \quad p_{2}=\lim _{(s=\infty)} s e^{-\lambda_{2} i}
$$

$\left(0 \leqq \lambda_{1}<\frac{\pi}{2}, 0 \leqq \lambda_{2}<\frac{\pi}{2}\right)$ gesetzt ist, und unter $\mathfrak{p}^{\prime}$ ein beliebiger der zwei Werthe $p_{1}, p_{2}$ verstanden wird. Der Integrationsweg von (12) wird in Figur 3 durch $p_{1} p_{1} q p_{2} p_{2}$,

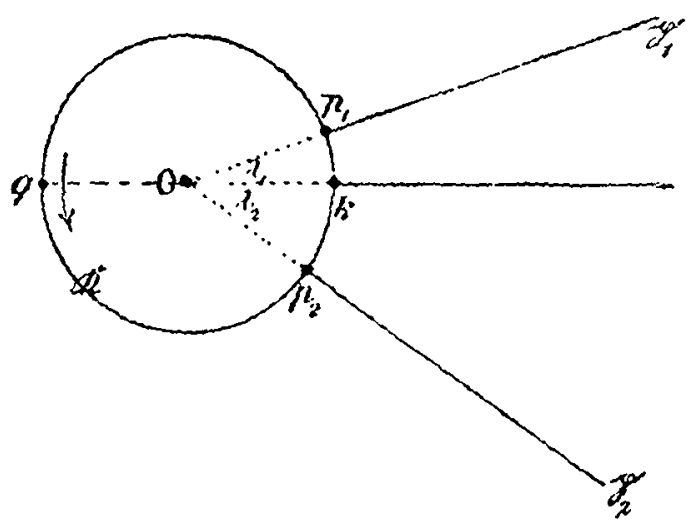

Fig. 3. der Integrationsweg yon (13) durch $p_{1} p_{1} q p_{2} k p_{1} p_{1}$ oder $p_{2} p_{2} k p_{1} q p_{2} p_{2}$ angegeben. Für die Fixirung des Zweiges der Potenz $u^{a-1}$ möge in (12) und (13) derjenige Punkt benutzt werden, in welchem der Integrationsweg die negative reelle Axe schueidet. Bei dem Integral (2) geht die Variable $u$ vom Punkte $k$ zum Punkte - $k$ (in Fig. 1, 2, 3 durch $q$ bezeichnet) über, indem sie die obere Hälfte des Kreises $\Re$ im positiven Sinue durchläuft. Daher hat $u^{a-1}$ für $u=-k$ den Werth $e^{(a-1)(\pi i+\log k)}$ wo $\log k$ den reellen Logarithmus bedeutet. Diese Bestimmung bleibt für die Integrale (12) und (13) in Kraft.

In ähnlicher Weise lässt sich der Integrationsweg der Integrale $(5),(6),(7)$ modificiren. Nennt man $\mathfrak{c}_{1}$ und $\mathfrak{c}_{2}$ die Producte

$$
\mathfrak{e}_{1}=\mathfrak{e} e^{-\left(\pi-\lambda_{1}\right) i}, \quad \mathfrak{e}_{2}=e e^{\left(x-\lambda_{2}\right) i},
$$

in denen $e$ wiederum eine unendlich kleine positive reelle $\mathrm{Zahl}$ bezeichnet, und $\lambda_{1}, \lambda_{2}$ reell, positiv und kleiner als $\frac{\pi}{2}$ sind, so kann man aach Fig. 2 in (5) den Weg

$$
\mathrm{e}_{1} m_{1} i_{1} k i_{2} m_{2} \mathrm{c}_{2}
$$

oder auch einen der zwei Wege

$$
\mathfrak{e}_{2} m_{1} i_{1} k i_{2} m_{2} q m_{1} \mathfrak{e}_{1}, \quad \mathfrak{e}_{2} m m_{2} q m_{1} i_{1} k i_{2} m_{2} \mathfrak{e}_{2}
$$

für die Variable $t$ wählen, ohne hierduxch den Werth des Integrals (5) zu ändern. Denn das Integral der Function $e^{\frac{1}{t}} t^{-a-1}$, genommen längs eines unendlich kleinen Kreisbogens (mit dem Radius $\mathfrak{c}$ ), welcher voll - $\mathfrak{c}$ bis $\mathfrak{e}_{1}$ oder von $-\mathfrak{e}$ bis $\mathfrak{k}_{2}$ (auf der Seite der negativen reellen Werthe) führt, ist eine unendlich kleine Grösse. Demnach werden aus (3) die weiteren Formeln 


$$
\vec{\Gamma}(a)=\int_{\mathrm{e}_{1}}^{\mathrm{e}_{2}} e^{\frac{1}{t}} t^{-a-1} d t
$$

und

$$
\bar{\Gamma}(a)=\bar{\int}_{\mathfrak{c}^{\prime}}^{(0)} e^{\frac{1}{t}} t^{-a-1} d t
$$

erhalten, in denen die soeben angeführten Integrationswerge anzuwenden sind, und durch $\mathfrak{c}^{\prime}$ ein beliebiger der zwei Werthe $c_{1}$, $c_{\text {.. bezcichet }}$ wird. Für $t=k$ (Fig. 2) hat man in den beiden letzten (ileichungen die Potenz $t^{-a-1}$ gleich demjenigen Werthe $e^{-(a+1)_{0} k}$, in welchem $\log k$ reell ist, zu nehmen.

\section{$\S 3$.}

Es soll noch kurz auf den Fall eingegangen werden, diss in den vorstehenden Rechnungen die Constanten $\lambda_{1}$ and $\lambda_{2}$ den Werth ${ }_{2}$ annehmen, so dass in Fig. 2 die Geraden $\dddot{M}_{1}$ und $\|_{i}$, in Nen negativen und den positiven $\mathrm{Zweig}$ der imaginären Axe iibergehen. In $\$$ : wurde mit Hülfe der Ungleichheit (8) gezeigt, dass das Integral dor function $e^{u} u^{a-1}$ einen unendlich kleinen Werth hat, wemn dic Integration iiber einen (bei $-\infty$ beginnenden) Theil des zwischen - - $\infty$ und - - $\infty$, resp. zwischen $-\infty$ und $+\infty i$ liegenden Quadranten des unendlichen Horizonts erstreckt wird. Nun verschwindet, wie aus (X) folgt, auch las über den ganzen Quadranten ausgedehnte Integral (was in (8) dem Werth $\lambda=\frac{\pi}{2}$ entspricht), falls der reelle 'Theil $a_{1}$ der Constanto a negativ ist. Allerdings darf für $\lambda=\frac{\pi}{2}$ die auf der rechten Seito von (8) stehende Exponentialgrösse $e^{s \cos \vartheta_{0}-a_{2} \vartheta_{0}}$ mit wachsendem s nicht mehr als identisch mit Null angenommen werden, da in Berug auf $\boldsymbol{\theta}_{0}$ nur die Grenzwerthe $-\pi$ und $-\frac{\pi}{2}$ (in $\S 2:-\pi+\lambda$ ), \%wischen denen $\vartheta_{0}$ liegt, bekannt sind, und folglich für $\cos \vartheta_{0}$ der Werth Nuli nicht ausgeschlossen ist. Im Falle $a_{1}<0$ verschwiudet aber lie rechte Seite von (8) für $s=\infty$ wegen des Factors $s^{a_{1}}$.

Diese Betrachtungen führen zu dein Grenztall der form.! (!), w! $\mathfrak{m}_{1}$ durch $-\infty i$ und $\mathfrak{m}_{2}$ durch $+\infty i$ ersetzt wird. Mun hat., wenn der reelle Theil von a negativ ist, die Gleichung

$$
\bar{r}(a)=\int_{-\infty i}^{+\infty i} e^{u} u^{a-1} d u
$$

in welcher die Variable $u$ im Uebrigen die ganze inarginïre Axe durchläuft, jedoch den Nullpunkt auf der Seite der positiven reellen Werthe umgeht. Denn die Schlüsse, durch die mau die Gileichuug (9) bewies, 
bleiben auch hier in Kraft. Der Integrationsweg von (18) besteht nach Fig. 2 aus den ausserhalb der Punkte $i_{1}$ und $i_{2}$ liegenden Abschnitten der imaginären $A x e$ und aus dem Halbkreise $i_{1} k i_{2}$. Dagegen ist

$$
\int_{-\infty i}^{+\infty i} e^{u} u^{a-1} d u=0
$$

falls der Weg der Variablen $u$ statt des Halbkreises $i_{1} k i_{2}$ den Halbkreis $i_{1} q i_{2}$ (Fig. 2) enthält, f'alls also der Nullpunkt auf der Seite der negativen reellen Werthe umgangen wird. Die geschlossene Curve, welche sich aus der geradlinigen Strecke von $-\infty i$ bis zum Punkte $i_{1}$, dem Halbkreise $i_{1} q i_{2}$, der geradlinigen Strecke von $i_{2}$ bis $+\infty i$ und der auf der Seite der negativen reellen Werthe liegenden Hälfte des unendlichen Horizonts zusammensetzt, bildet die Begrenzung eines Flächenstücks, auf dem die Function $e^{u} u^{a-1}$ eindeutig und stetig ist. Da nun das über die Horizonthälfte erstreckte Integral den Werth Null hat, so verschwindet auch das Integral (19), dessen Integrationsweg aus deu drei übrigen genannten Strecken besteht.

Substituirt man in (19) $u=-v$, so entsteht die Gleichung

$$
\int_{-\infty i}^{+\infty i} e^{-v} v^{a-1} d v=0
$$

in welcher vorausgesetzt ist, dass die Variable den Nullpunkt auf der Seite der positiven reellen Werthe umgeht.

Wie die Formel (9), so kann auch die Formel (11), sobald der reelle Theil von a negativ ist, in der Art modificirt werden, dass der Werth $-\infty i$ an die Stelle ron $m_{1}$, resp. der Werth $+\infty i$ an die Stelle von $\mathfrak{m}_{2}$ tritt. Man erhält, gemäss den Bemerkungen am Eingang dieses Paragraphen, im Fall $a_{1}<0$ die Gleichungen

$$
\begin{aligned}
& \bar{\Gamma}(a)=\int_{-\infty i}^{\bar{*}(0)} e^{u} u^{a-1} d u, \\
& \bar{\Gamma}(a)=\int_{+\infty i}^{-\infty(0)} e^{u} u^{a-1} d u .
\end{aligned}
$$

Hier kommt (nach Fig. 2) der ganze Kreis $\Omega$ in dem Integrationswege vor; ausserdem wird in (20) die Strecke von $-\infty i$ bis $i_{1}$, in (21) die Strecke von $+\infty i$ bis $i_{2}$ in beiden Richtungen durchlaufen.

Es möge erwähnt sein, dass die Gleichungen (18) und (19) gültig bleiben, wenn $a$ ein positiver reeller Bruch ist. Dieselben sind dann eine unmittelbare Folge der Dirichlet'schen Formeln

$$
\begin{aligned}
& \int_{0}^{\infty} w^{a-1} \cos w d w=\cos \frac{\pi a}{2} \Gamma(a), \\
& \int_{0}^{\infty} w^{a-1} \sin w d w=\sin \frac{\pi a}{2} \Gamma(a),
\end{aligned}
$$


in denen $\Gamma(a)$ das Euler'sche Integral $2^{\text {ter }}$ Art bedeutet. Bei dem Integral (18) nimmt die Potenz $u^{a-1}$ im Punkte $i_{1}$ (Fig. 2) den Werth $e^{-\frac{\pi i}{2}(a-1)} k^{a-1}$, im Punkte $i_{2}$ den Werth $e^{\frac{\pi i}{2}(a-1)} k^{a-1}$ an, wo $k^{a-1}$ die positive reelle Potenz von $k$ bedeutet. Denn nachdem $u=k e^{\vartheta i}$ gesetzt ist, hat man die anfangs erwähnte Bedingung für $u^{a-1} \mathrm{zu}$ berücksichtig en. Substituirt man auf der negativen imaginären Axe $u=-i r$, auf der positiven $u=+i r$, so wird auf ersterer das Product $u^{a-1} d u$ gleich $e^{-\frac{\pi i a}{2}} r^{a-1} d r$, auf letzterer gleich $e^{\frac{\pi i a}{2}} r^{a-1} d r$, da die Bestim. mung im Punkte $i_{1}$, resp. $i_{2}$, massgebend ist. Bei dem Integral (19) hat dagegen, weil der Nullpunkt auf der negativen Seite umgangen wird, das Product $u^{a-1} d u$ auf der positiven imaginären Axe den Werth $e^{-\frac{3 \pi i a}{8}} r^{a-1} d r$.

lich klein; dann sind die Integrale der Function $c^{u} u^{a-1}$ längs der Halbkreise $i_{1} k i_{2}$ und $i_{1} q i_{2}$ (da a positiv sein soll) zu vernachlässigende Grössen. Hierdurch geht das auf der rechten Seite von (18) stehende Integral, wenn man die Gleichungen (22) und (23) benutzt, in die Summe

$$
\begin{gathered}
\left(e^{\frac{\pi i a}{2}}-e^{-\frac{\pi i a}{2}}\right) \int_{0}^{\infty} r^{a-1} \cos r d r+i\left(e^{\frac{\pi i a}{2}}+e^{\left.-\frac{\pi i a}{2}\right)} \int_{0}^{\infty} r^{a-1} \sin r d r\right. \\
=2 i \sin (\pi a) \Gamma(a)=\bar{\Gamma}(a)
\end{gathered}
$$

über, während für das Integral (19) der Werth

$e^{-\pi i a} \Gamma(a)\left\{-\cos \frac{\pi a}{2}\left(e^{\frac{\pi i a}{2}}-e^{-\frac{\pi i a}{2}}\right)+i \sin \frac{\pi a}{2}\left(e^{\frac{\pi i a}{2}}+e^{-\frac{\pi i a}{2}}\right)\right\}=0$ erhalten wird.

Formt man das Integral (18) durch die Substitution $u=\frac{1}{t} \mathrm{um}$, so ergiebt sich, dass $\Gamma(a)$ auch mit dem Ausdruck

$$
\bar{\Gamma}(a)=\int_{-e i}^{+e i} e^{\frac{1}{t}} t^{-a-1} d t
$$

identisch ist, falls die Variable $t$ den Nullpunkt auf der Seite der positiven reellen Werthe umgeht. Durch $c$ wird, wie in $\S 1$, eine unendlich kleine positive reelle Constante bezeichnet. Der Integrationsweg von (24) lässt sich (da $k$ willkürlich ist, statt $\frac{1}{l_{6}}$ also wieder $k$ genommen werden darf) nach Fig. 2 durch das Stück der negativen imaginären Axe von 0 bis $i_{1}$, den Halbkreis $i_{1} k i_{2}$ und das Stück der positiven imaginären Axe von $i_{2}$ bis 0 darstellen. Wird der genannte Weg in der Art abgeändert, dass der Halbkreis $i_{1} q i_{2}$ an die Stelle des Halbkreises $i_{1} k i_{2}$ tritt, so ist, wie aus (19) folgt, 


$$
\int_{-t i}^{+e i} e^{\frac{1}{t}} t^{-a-1} d t=0 .
$$

Aus (20) und (21) erhält man, wenn $u=\frac{1}{t}$ gesetzt wird, die zu (17) analogen Gleichungen

$$
\bar{\Gamma}(a)=\int_{-i i}^{(0)} e^{\frac{1}{t}} t^{-a-1} d t
$$

und

$$
\bar{\Gamma}(a)=\int_{e i}^{(0)} e^{\frac{1}{t}} t^{-a-1} d t .
$$

In den Formeln (24) bis (27) wird der reelle Bestandtheil der Constante $a$ als negativ vorausgesetzt. Der in (24), (26), (27) anzuwendende Zweig der Potenz $t^{-a-1}$ ist derselbe wie in (5), (16), (17), d. h. es gilt auch hier für $t^{-a-1}$ die am Schlusse des $\$ 2$ genannte Bedingung.

Kiel, im Mai 1891. 